Research Paper

\title{
Secretory Clusterin Mediates Oxaliplatin Resistance via the Gadd45a/PI3K/Akt Signaling Pathway in Hepatocellular Carcinoma
}

\begin{abstract}
Xin Wang ${ }^{1 *}$, Fang Zou ${ }^{1,2^{*}}$, Jingtao Zhong ${ }^{3}$, Longtao Yue ${ }^{1}$, Fuhai Wang ${ }^{1}$, Honglong Wei ${ }^{1}$, Guangsheng Yang ${ }^{1}$,
\end{abstract} Tao Jin ${ }^{1}$, Xiaofeng Dong ${ }^{4}$, Jie Li ${ }^{1}$, Peng $\mathrm{Xiu}^{1,}{ }^{\otimes}$

1. Department of General Surgery, Shandong Provincial Qianfoshan Hospital, Shandong University, Jinan 250014, China

2. Department of Emergency Surgery, The People's Hospital of Linyi City, Linyi 276000, China

3. Department of General Surgery, Shandong Cancer Hospital Affiliated to Shandong University, Shandong Academic of Medicine Science, Jinan 250117, Shandong, China

4. Department of Hepatobiliary Surgery, The People's Hospital of Guangxi Zhuang Autonomous Region, Nanning 530021, China

* Xin Wang and Fang Zou are equal contributors to this paper.

$\bowtie$ Corresponding author: Peng Xiu, Tel/Fax: +86-531-89269815. E-mail address: Lcxiupeng@126.com.

(c) Ivyspring International Publisher. This is an open access article distributed under the terms of the Creative Commons Attribution (CC BY-NC) license (https://creativecommons.org/licenses/by-nc/4.0/). See http://ivyspring.com/terms for full terms and conditions.

Received: 2017.11.14; Accepted: 2018.02.25; Published: 2018.04.06

\begin{abstract}
Purpose: Systemic therapy has often been used for patients with advanced hepatocellular carcinoma (HCC). However, due to drug resistance, the use of cytotoxic chemotherapy in the treatment of patients with advanced HCC has typically demonstrated low response rates. Secretory clusterin (sCLU) is expressed in aggressive late-stage tumors and associated with resistance to chemotherapy, including that in HCC cases. The present research aimed to investigate the biological role of $s \mathrm{CLU}$ in HCC.

Methods: $\mathrm{sCLU}$ expression in $\mathrm{HCC}$ and normal tissues was examined using immunohistochemical staining, followed by analysis of the correlation between sCLU expression and clinical indicators. In addition, the role and internal mechanism of $\mathrm{sCLU}$ in cell proliferation and apoptosis were investigated in HCC cells.

Results: sCLU expression was significantly upregulated in HCC tissues; and was associated with histological grade and poor overall survival. The levels of SCLU were significantly increased in Bel7402, SMMC7721 and resistant HCC cells (Bel7404-OR). Inhibiting the activity of sCLU enhanced the chemosensitivity of Bel7402 and SMMC7721 cells. Downregulation of sCLU could increase the expression of Gadd45a in HCC cells. Overexpression of sCLU contributed to drug resistance in Bel7402, SMMC7721 and Bel7404-OR cells; whereas, overexpression of Gadd45a alone overcame drug resistance in the cells above. No significant expression changes of $s C L U$ and Gadd45a were observed in HCC cells after the interference of a selective inhibitor of the PI3K/Akt signaling pathway. However, regulation of the expression of Gadd45a could influence the phosphorylation level of Akt; and further regulate the expression of $\mathrm{Bcl}-2$ and $\mathrm{Bax}$ proteins involved in the mitochondrial apoptosis pathways.
\end{abstract}

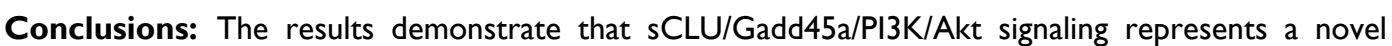
pathway that could regulate drug resistance in a one-way manner in HCC cells.

Key words:

\section{Introduction}

It is widely known that liver cancer rates are decreasing in China due to reductions in HBV infection because of improved hygiene and sanitation[1]. However, liver cancer, which is known as HCC and occurs world-wide, is still the fourth most common cancer and third major reason for death caused by cancer in China[2]. Until very recently, no systemic chemotherapy was shown to be effective or 
continuous in the treatment of HCC diagnosed at advanced stages or for those who progressed into an advanced stage after other treatments failed because HCC is extremely resistant to current chemotherapeutic drugs. Sorafenib[3], an oral multikinase inhibitor that suppresses tumor cell proliferation and angiogenesis, is the first FDA-approved systemic therapy for patients with advanced HCC. However, it offers limited survival benefits; and is hampered by the occurrence of drug resistance and tumor relapse[4]. Therefore, the present status of this treatment has instigated intense research on drug resistance and molecular mechanisms of HCC with the hope of developing new treatment strategies.

CLU is a single-copy gene, located on chromosome 8p21-p12, where it is organized into nine exons, eight introns and a 5 '-untranslated region and encodes three different transcriptional isoforms in humans[5]. Two proteins are encoded by this gene: secretory CLU protein (sCLU) $(75-80 \mathrm{kDa})$ and nuclear CLU protein (nCLU) (55 kDa) [6, 7]. In many human tumors, sCLU, which is an endoplasmic reticulum (ER)-targeted 449-amino acid polypeptide, is considered a stress-activated, cytoprotective, and small heat shock chaperone that has been defined as an anti-apoptotic protein[8]. Despite growing evidence that sCLU, which is an anti-apoptotic factor, plays an important role in the resistance to chemotherapeutic drugs[9, 10], the molecular mechanism involved in this process remains unclear[11].

Previous studies have proven that the constitutive activation of the PI3K/AKT/mTOR signaling pathway was a major determinant of tumor cell growth, survival and drug resistance[12]. Our previous results indicate that sCLU contributes to OXA resistance by activating the PI3K/Akt pathway[13]. Regulating sCLU expression could influence the expression of Gadd45a, which is considered an essential step following stressful growth arrest conditions and treatment with pro-apoptotic agents[14]. In HCC cells, whether Gadd45a can play an important role in the sensitization process of sCLU; and whether it has upstream and downstream control relationships with the PI3K/AKT signaling pathway remain unknown problems. Therefore, we designed the present study to investigate the molecular mechanisms of sCLU in regulating the downstream signaling pathways.

\section{Methods}

Cell lines and culture. Bel7402, Bel7404, and SMMC7721 cells (human hepatocellular carcinoma cell lines) were cultured in RPMI-1640 (Thermo Fisher
Scientific, Shanghai, China) supplemented with 100 $\mathrm{U} / \mathrm{ml}$ penicillin, $100 \mathrm{mg} / \mathrm{ml}$ streptomycin, and $10 \%$ fetal bovine serum. The cells were obtained from the Type Culture Collection of the Chinese Academy of Sciences (Shanghai, China). All cultures were grown at $37^{\circ} \mathrm{C}$ in a humidified atmosphere containing $5 \%$ carbon dioxide[13]. The OXA (oxaliplatin)-resistant cells, termed Bel7404-OR, were established by incubating Bel7404 cells with OXA[13].

Antibodies and reagents. The antibodies used in this study included an antibody against clusterin (\#42143), which was obtained from Cell Signaling Technology (Danvers, MA, USA), and antibodies against GADD45a (ab180768), AKT1 (ab18206), AKT (ab8805), Bcl-2 (ab32124) and Bax (ab32503), which were obtained from Abcam (Cambridge, UK). The PI3-kinase inhibitor (LY294002) (ab120243) was purchased from Abcam (Cambridge, UK).

IHC and histopathological analyses. Human hepatocellular carcinoma tissue arrays were obtained from Shanghai Biochip Company Ltd. (Shanghai, China). HCC tissues were obtained from 105 HCC patients undergoing complete surgical resection between January 2007 and November 2009. All patients were followed up until September 2013. None of the patients received radiotherapy or chemotherapy before surgery, and none of them had multiple cancers in other organs.

The tissue microarray was deparaffinized, rehydrated through graded alcohol series, washed with Tris-buffered saline, and processed using a streptavidin-biotin-peroxidase complex method. The tissue microarray was incubated with mouse anti-Clusterin (1:200, Cell Signaling Technology Danvers, MA, USA) overnight at $4^{\circ} \mathrm{C}$, followed by incubation with horseradish peroxidase-conjugated secondary antibody. Next, the tissue microarray was assessed by two pathologists who were blinded to the clinical and pathologic information. The staining intensity of the tumor tissues was scored as 0 (negative), $1+$ (weak), $2+$ (moderate) or $3+$ (strong), while the staining extent was scored as $0(<10 \%), 1+$ $(10 \%-25 \%), 2+(26 \%-50 \%)$ or $3+(>50 \%)[15,16]$. The product of staining intensity by the staining extent was defined as the final staining score which was classified as negativity (score $0-3$ ) and positivity (score 4-9)[17].

Plasmid construction and transfection, cell viability and apoptosis assays. Bel7404 cells stably transfected with the pIRES2-EGFP/sCLU and pIRES2-EGFP vectors were termed Bel7404-sCLUhigh and Bel7404-vec, respectively. Bel7402 or SMMC7721 cells stably transfected with sCLU shRNA (CLU-4) or Sc vectors were termed Bel7402-sCLUlow, Bel7402-vec, SMMC7721-sCLUlow or SMMC7721-vec. The Gadd45a 
expression vector was constructed and termed pcDNA3.1-Gadd45a-His. The plasmids were transfected into Bel7402 and SMMC7721 cells and were termed as Bel7402-Gadd45a and SMMC7721-Gadd45a, respectively. All these methods were described previously[13, 14].

Western blot analysis for protein expression. Whole proteins from cultured HCC cells were extracted using a protein extraction kit (Beyotime, Shanghai, China). In total, $50 \mu \mathrm{g}$ of proteins was resolved on an $8 \%$ SDS-PAGE gel and was transferred to a PVDF membrane (Millipore). Non-specific binding sites in the membranes were blocked with $1 \%$ bovine serum albumin in TBST buffer for $2 \mathrm{~h}$ at room temperature before the addition of primary antibodies. Appropriate anti-rabbit, anti-mouse and anti-goat antibodies were used as secondary antibodies for $2 \mathrm{~h}$ at $37^{\circ} \mathrm{C}$. Protein bands were detected by using an ECL detection kit (Millipore, MA, USA) and protein expression was determined using Quantity One software and was normalized to GAPDH.

Statistical analysis. All data were expressed as the mean \pm standard deviation (SD) and were analyzed by SPSS 17.0 software. Quantitative data were analyzed using one-way ANOVA. Survival curves were evaluated using the Kaplan-Meier method and were compared using the log-rank test. A value of $p<0.05$ was considered significant.

\section{Results}

\section{Negative sCLU expression correlates with better survival rates in $\mathrm{HCC}$}

In total, 105 HCC patients were included in this study, and the clinical characteristics are summarized in Table 1. The association between sCLU expression and clinicopathological characteristics of the HCC patients was analyzed. According to the IHC results, 63 cases $(60.0 \%)$ exhibited higher expression of sCLU in HCC specimens, whereas only 19 cases $(18.1 \%)$ of the matched non-tumorous tissue samples demonstrated positive sCLU expression $(\mathrm{P}<0.001)$ (Fig. 1A and 1B).

Next, we examined the prognostic value of sCLU for overall survival in HCC. Kaplan-Meier survival curves revealed that the HCC patients with low expression of sCLU survived significantly longer than those with high expression of sCLU (Fig. 1C). The mean survival time of patients with negative sCLU expression was 54.0 months, but it decreased to 18.0 months in patients with positive sCLU expression. We could also find that tumor stage was a prognostic factor (Fig. 1D). All these results demonstrated that negative sCLU expression was significantly associated with better overall survival.

Table 1. Correlation between the expression of sCLU and the clinicopathological characteristics of HCC patients

\begin{tabular}{|c|c|c|c|c|}
\hline \multirow[t]{2}{*}{ Variables } & \multirow[t]{2}{*}{$\mathrm{N}$} & \multicolumn{2}{|c|}{ sCLU Expression } & \multirow[t]{2}{*}{$P$} \\
\hline & & Negative & Positive & \\
\hline Age(years) & & & & 0.322 \\
\hline$\leq 55$ & 66 & 24 & 42 & \\
\hline$>55$ & 39 & 18 & 21 & \\
\hline Gender & & & & 0.298 \\
\hline Male & 94 & 36 & 58 & \\
\hline Female & 11 & 6 & 5 & \\
\hline Tumor size $(\mathrm{cm})$ & & & & 0.128 \\
\hline$\leq 2$ & 14 & 3 & 11 & \\
\hline$>2$ & 91 & 39 & 52 & \\
\hline Tumor size $(\mathrm{cm})$ & & & & 0.568 \\
\hline$\leq 5$ & 41 & 15 & 26 & \\
\hline$>5$ & 64 & 27 & 37 & \\
\hline Metastasis & & & & 0.255 \\
\hline Yes & 9 & 2 & 7 & \\
\hline No & 96 & 40 & 56 & \\
\hline HBsAg & & & & 0.255 \\
\hline Positive & 81 & 30 & 51 & \\
\hline Negative & 24 & 12 & 12 & \\
\hline $\begin{array}{l}\text { Histological } \\
\text { grades }\end{array}$ & & & & $0.046^{*}$ \\
\hline $\mathrm{I}+\mathrm{II}$ & 55 & 27 & 28 & \\
\hline III & 50 & 15 & 35 & \\
\hline TNM stage & & & & 0.262 \\
\hline $\mathrm{I}+\mathrm{II}$ & 58 & 26 & 32 & \\
\hline $\mathrm{III}+\mathrm{IV}$ & 47 & 16 & 31 & \\
\hline
\end{tabular}

*Statistically significant of $\mathrm{P}<0.05$

\section{Relationship between sCLU expression and Gadd45a expression in HCC cells.}

Our previous study has demonstrated that down-regulated expression of sCLU results in a significant increase in Gadd45a protein levels[13, 14]. However, the role of Gadd45a and its downstream signaling pathways in the chemosensitization of sCLU require further research. Hence, we investigated the expression of sCLU and Gadd45a by Western blotting in HCC cells. As shown in Fig. 2A, compared with Bel7404 cells, which showed a low expression of sCLU protein, there was a low level Gadd45a expression in Bel7402 and SMMC7721 cells with high expression of sCLU protein.

To analyze the expression of Gadd45a, we silenced the sCLU gene using shRNA in Bel7402 and SMMC7721 cells and increased the expression of sCLU using the pIRES2-EGFP/sCLU in Bel7404 cells. As shown in Fig. $2 \mathrm{C}$ and 2E, compared with the Bel7402-vec and SMMC7721-vec cells, the Gadd45a protein expression levels were increased in SMMC7721-sCLUlow and Bel7402-sCLUlow cells. However, there were no obvious differences in Gadd45a expression in Bel7404-sCLUhigh and Bel7404-vec cells (Fig. 2G and 2H). 

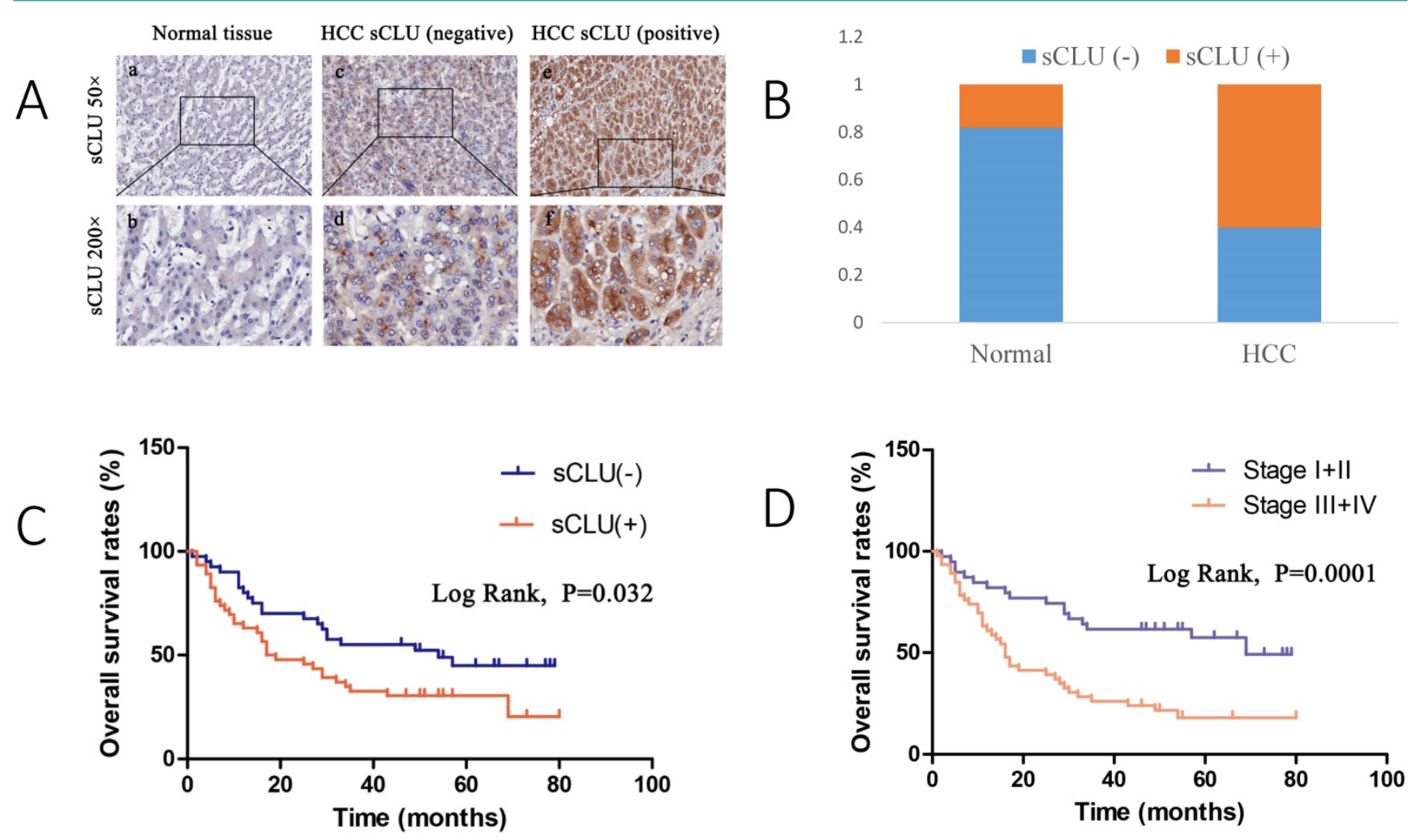

Fig. 1. sCLU expression is elevated and correlated with a poor prognosis in HCC. A. Immunohistochemical analysis of sCLU expression in HCC tissues and adjacent nontumor liver tissues (50x and 200x). (a, b) Negative expression of sCLU in normal tissues; (c, d) Negative expression of sCLU in HCC tissues; (e, f) Positive expression of sCLU in $\mathrm{HCC}$ tissues. B. Percentage of sCLU immunostaining in HCC and normal tissues. C. Kaplan-Meier curves according to the sCLU expression status in HCC. D. Kaplan-Meier curves according to TNM stage in HCC. Patients at stage III+IV have shorter overall survival time than those at stage I+II.

\section{Regulation of the expression of sCLU and Gadd45a increases the sensitivity of Bel7402 and SMMC7721 cells to OXA}

Our previous research showed that there was significant overexpression of sCLU in Bel7402 and SMMC7721 cells, as well as a downregulation of sCLU expression that increased the sensitivity of HCC cells to OXA. However, the role of Gadd45a in the process remains unclear. The overexpression effect was confirmed by Western blotting in Bel7402 and SMMC7721 cells. Fig. 3A shows that the expression level of Gadd45a was significantly increased in Bel7402-Gadd45a and SMMC7721-Gadd45a cells compared with that in control transfectants.

Next, the dose at which OXA cytotoxicity develops, in the period of $24 \mathrm{~h}$ or $48 \mathrm{~h}$ in Bel7402 and SMMC7721 cells was determined. Cell viability was significantly decreased by OXA in a dose-dependent manner (Fig. 3C and 3D). Fig. 3E and $3 F$ shows that the depletion of sCLU or overexpression of Gadd45a could reduce the viability of Bel7402 and SMMC7721 cells treated with OXA $(16 \mu \mathrm{M})$, compared with the cells treated with OXA alone. Depletion of sCLU and overexpression of Gadd45a induced the apoptosis of Bel7402 cells at rates of $2.2 \%$ and $2.5 \%$, respectively. Oxaliplatin induced apoptosis at a rate of $8.8 \%$, which was significantly higher than that of untreated Bel7402 cells (0.7\%). Bel7402-sCLUhigh and Bel7402-Gadd45a cells treated with OXA had even higher apoptosis rates (Fig. 3G), while similar results were also obtained with SMMC7721 cells (Fig. 3H).

\section{Overexpression of Gadd45a increases the chemotherapy sensitivity of Bel7404-OR cells to OXA}

After evaluating the effect of sCLU gene suppression and Gadd45a overexpression in Bel7402 and SMMC7721 cells on tumor cell chemosensitivity, we next investigated the expression of sCLU and Gadd45a by Western blotting in the drug-resistant HCC cells (Bel7404-OR and Bel7404-OR-Gadd45a) and HCC cells with sCLU overexpression (Bel7404-sCLUhigh). As shown in Fig. 4A, compared with Bel7404 cells, sCLU expression was significantly upregulated in Bel7404-OR cells and Bel7404-OR-Gadd45a cells, with the highest expression level in Bel7404-sCLUhigh cells. After transfection with pIRES2-EGFP/Gadd45a in Bel7404-OR cells, these cells were expressed at distinctly higher levels of Gadd45a than parental cells. With the increased expression of sCLU in Bel7404-OR cells, we found that the expression of Gadd45a was decreased compared with that in the parental cells. 

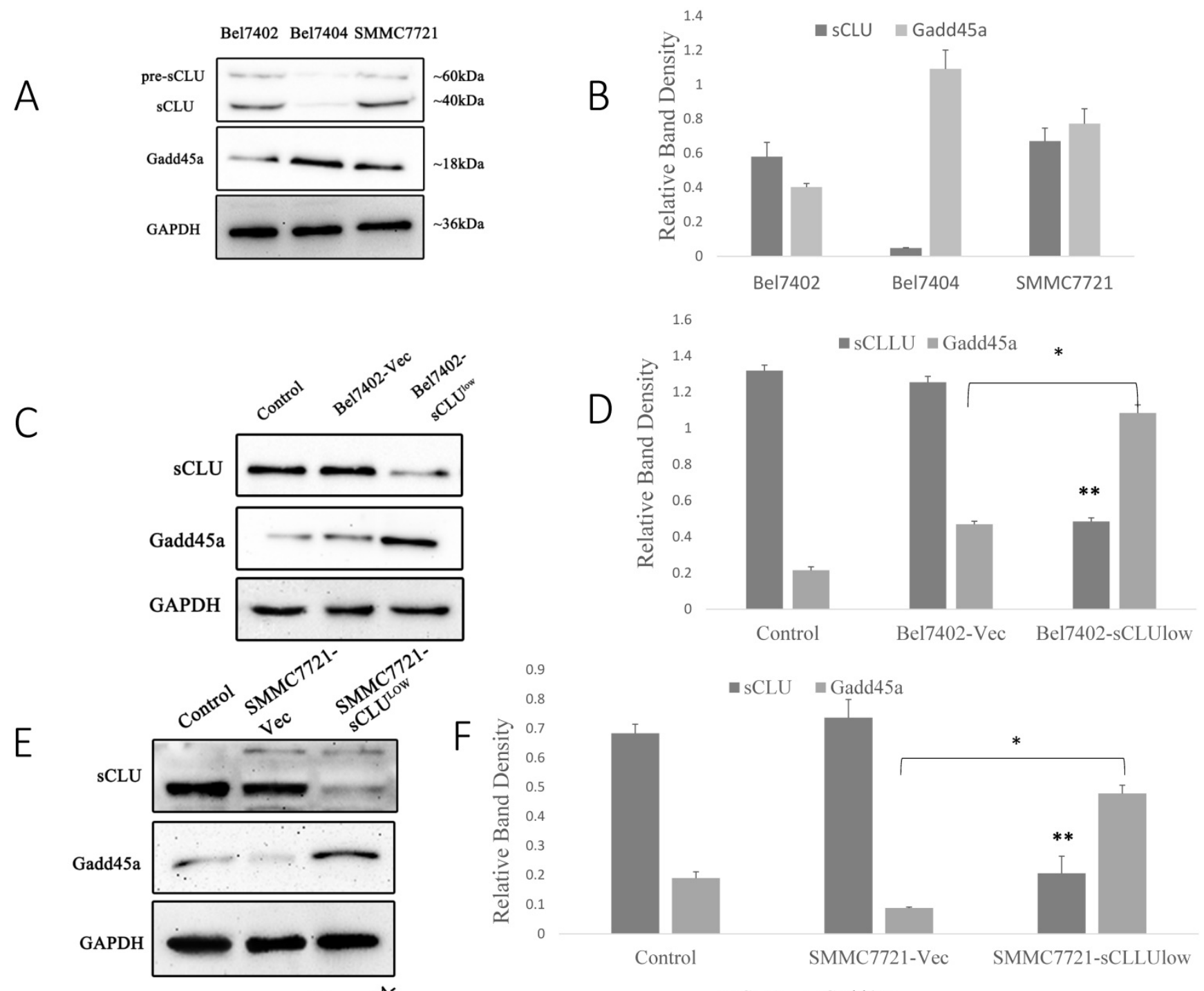

G
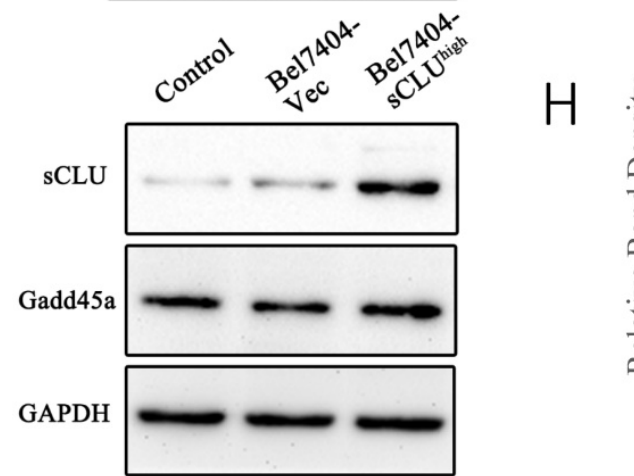

1.2
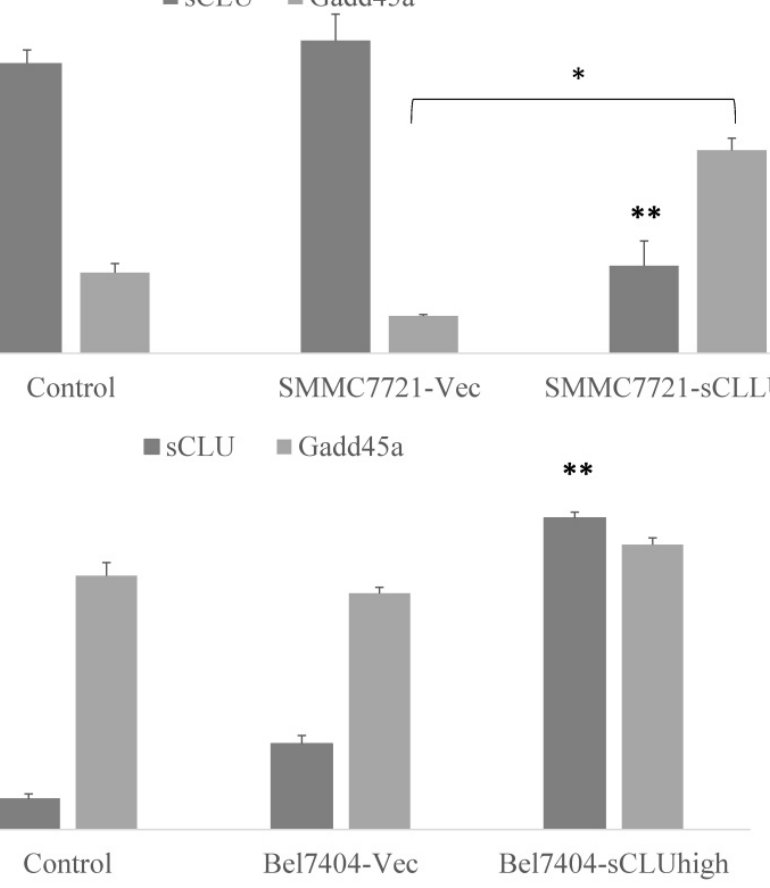

Fig. 2. Expression of sCLU and Gadd45a in HCC cells. Cell lysates (A) from SMMC7721, Bel7402 and Bel7404 cells were subjected to Western blot analysis. Parental Bel7402, SMMC7721 cells (Control), or Bel7402, SMMC7721 cells transfected with Sc or CLU-4 shRNA vectors were subjected to Western blot analysis (C and E). Parental Bel7404, or Bel7404 cells transfected with Sc or pIRES2-EGFP/sCLU vectors were subjected to Western blot analysis (G). (B, D F and H) The density of each band in (A, C, E and $\mathrm{G})$ was measured and normalized to that of GAPDH, respectively. A significant $(\mathrm{P}<0.05)$ difference of Gadd 45 a expression from the respective control is denoted by “*”, and sCLU expression is denoted by “**”.

According to Fig. 4C, with increasing concentrations of OXA, we found that the cell viability was higher in OXA-resistant Bel7404 cells (Bel7404-OR) than in parental Bel7404 cells. We also found the same results in Bel7404-sCLU cells. However, compared with Bel7404-OR cells, cell viability was significantly reduced in Bel7404-ORGadd45a cells (Fig. 4D). 

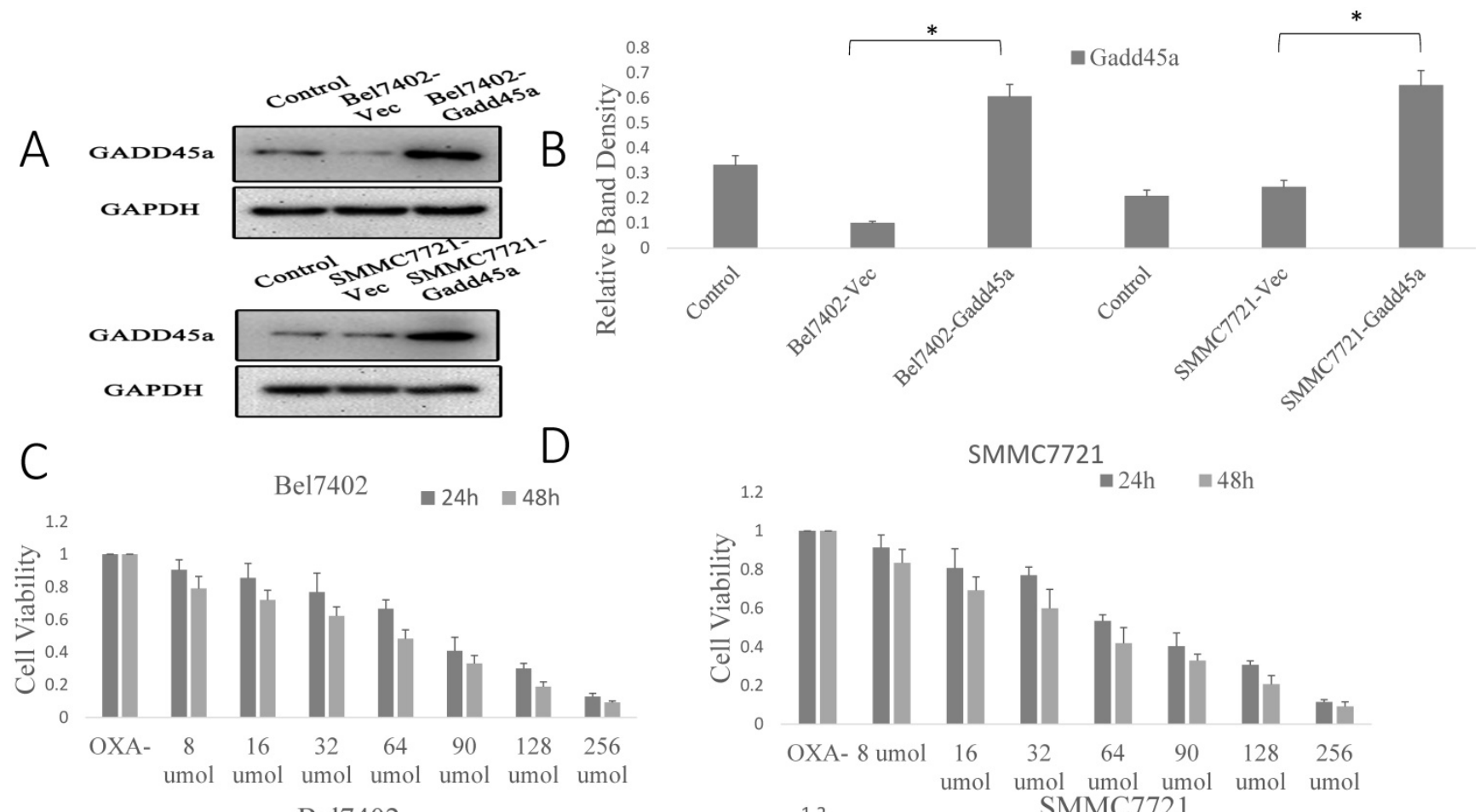

Bel7402
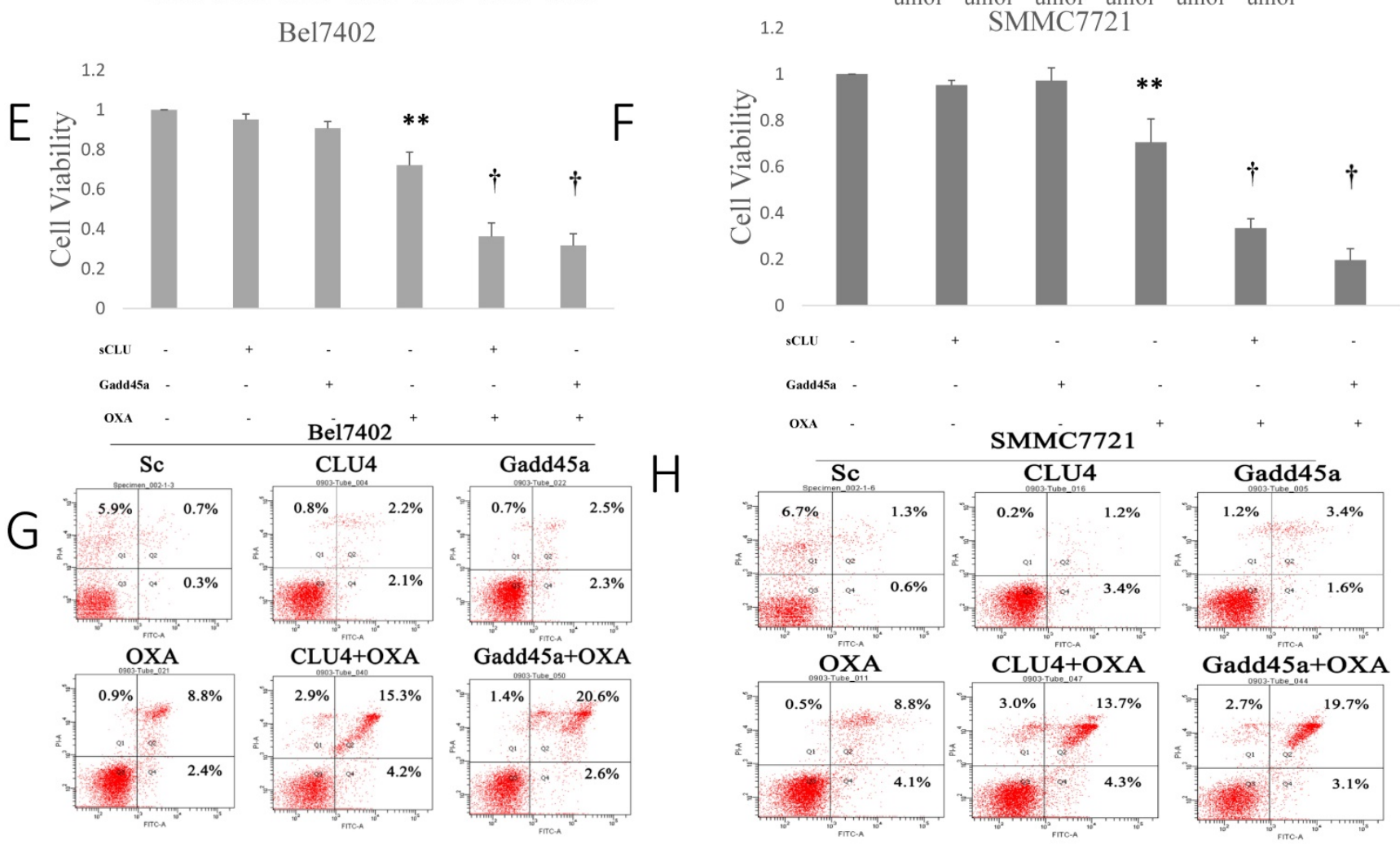

Fig. 3. Regulation of the expression of sCLU and Gadd45a increases the sensitivity of HCC cells to OXA. (A) Parental SMMC7721 and Bel7402 cells (Control), or cells transfected with Sc or pcDNA3.1-Gadd45a-His vectors were subjected to Western blot analysis. (B) The density of each band in (A) was measured and normalized to that of GAPDH. (C and D) Parental Bel7402 or SMMC7721 cells were seeded in a 96 -well plate and were allowed to grow overnight. The next day, the media were replaced with the same media containing various concentrations of oxaliplatin. The cell viability was measured using the CCK-8 assay after incubation for 24 and $48 \mathrm{~h}$. (E, F) Parental Bel7402 or SMMC7721 cells, cells transfected with CLU-4 shRNA vector, or cells transfected with pcDNA3.1-Gadd45a-His vectors were cultured in medium with $16 \mathrm{nM}$ OXA for $48 \mathrm{~h}$, and then the cell viability was measured. (G, H) Parental Bel7402 or SMMC7721 cells (control), cells transfected with CLU-4 or pcDNA3.1-Gadd45a-His vectors were incubated in the absence or presence of OXA $(16 \mathrm{nM})$ for $48 \mathrm{~h}$, and then the cell apoptosis rates(\%) were measured. A significant $(P<0.05)$ difference in the Gadd $45 \mathrm{a}$ expression from the respective control is denoted by “*”. A significant $(\mathrm{P}<0.05)$ reduction from OXA-treated parental cells is denoted by “***, and a significant $(\mathrm{P}<0.001)$ difference is denoted by “ $\dagger$ ". 


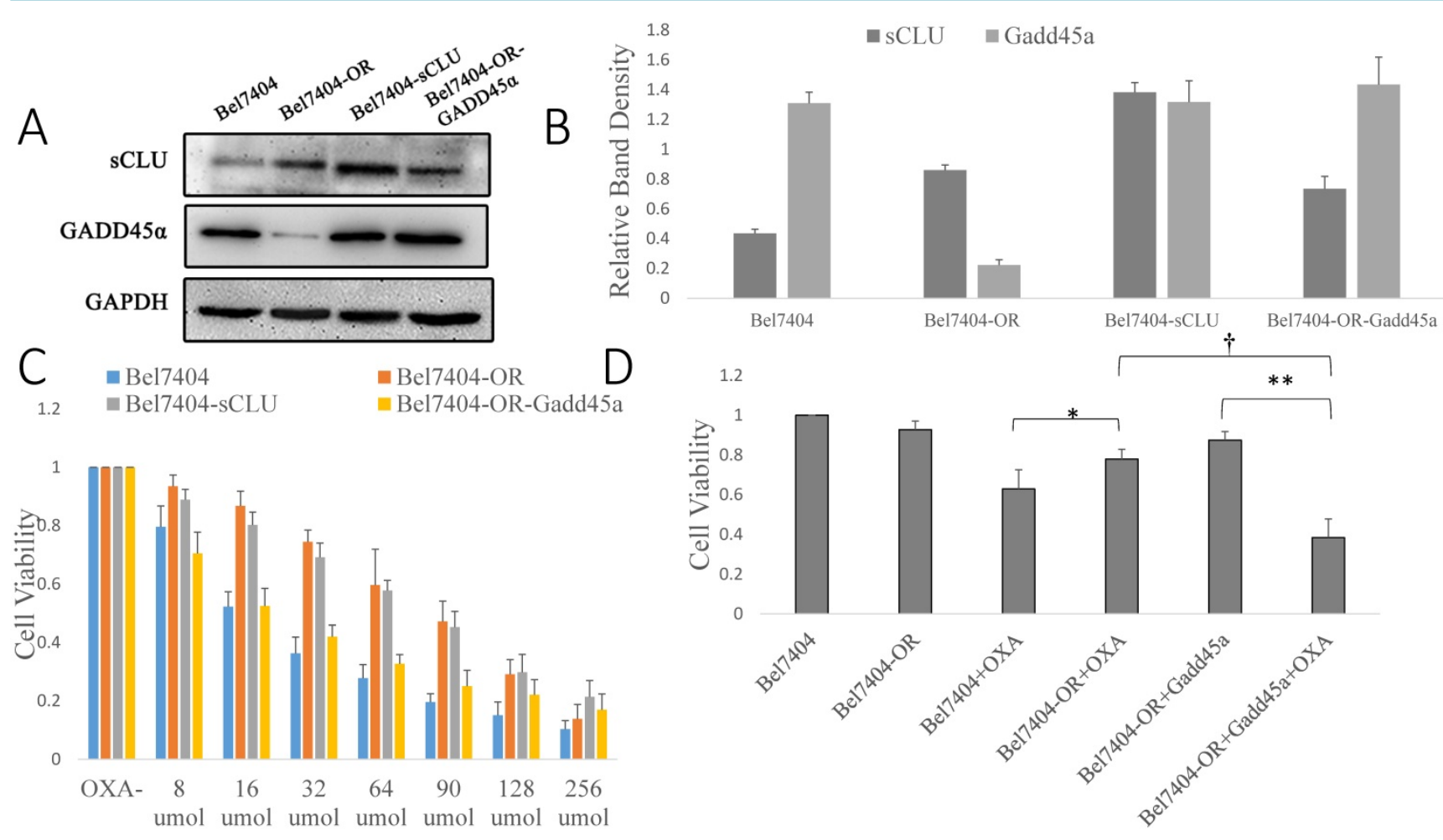

Fig. 4. Overexpression of the Gadd45a increases the chemotherapy sensitivity of Bel7404-OR cells to OXA. (A) Parental Bel7404 or Bel7404-OR cells, or cells transfected with pIRES2-EGFP/sCLU vectors, or pcDNA3.1-Gadd45a-His vectors, were subjected to Western blot analysis. (B) The density of each band in (A) was measured and normalized to that of GAPDH, respectively. (C, D) Parental Bel7404, Bel7404-OR cells, or cells transfected with pIRES2-EGFP/sCLU vectors or pcDNA3.1-Gadd45a-His vectors were seeded in 96-well plate and were allowed to grow overnight. The next day, the media were replaced with the same media containing various concentrations of OXA. After $48 \mathrm{~h}$ of incubation, the cell viability was measured using the CCK-8 assay. (D) The cells above were cultured in medium with $16 \mathrm{nM}$ OXA for $48 \mathrm{~h}$ and then the cell viability was measured. A significant $(\mathrm{P}<0.05)$ reduction in OXA-treated Bel7404 or Bel7404-OR cells is denoted by “*”, a significant reduction in Gadd45a overexpression cells is denoted by “***, and a significant difference in Gadd45a overexpression in Bel7404-OR cells is denoted by " $\dagger$ ".

\section{sCLU regulates the PI3K/AKT signaling pathway by mediating the expression of Gadd45a}

Early studies have shown that sCLU can regulate the PI3K/AKT signaling pathway, but it was unclear whether Gadd45a was involved in this process. pAkt has been considered a significant multidrug resistance locus and, thus, was emphasized to detect its expression in HCC cells. To further investigate the relationship among sCLU, Gadd45a and the PI3K/AKT pathway, we first explored the expression of sCLU and Gadd45a in HCC cells after the intervention of a PI3K/AKT-selective inhibitor. Western blot analysis showed that pAKT was downregulated and there were no differences in the expression of sCLU and Gadd45a in Bel7402 and SMMC7721 cells after treatment with LY294002, a potent inhibitor of PI3K (Fig. 5A and 5B). In addition, we obtained the same results in Bel7404 and Bel7404-OR cells (Fig. 5C and 5D). These data together implied that there were no significant differences in the expression of sCLU and Gadd45a after the inhibition of the PI3K/AKT pathway, while sCLU might regulate the PI3K/AKT pathway in a one-way control mode. To explore the role of Gadd45a in the process, the expression of pAkt, Bcl-2 and Bax was investigated in HCC cells after regulation of the expression of Gadd45a. We found that overexpression of Gadd45a could decrease the expression of pAkt in Bel7402, SMMC7721 and Bel7404-OR cells, while these cells had similar levels of Akt compared with those of their parents. In the same manner, Gadd45a overexpression upregulated Bax and downregulated $\mathrm{Bcl}-2$, thus reducing the Bcl-2/Bax ratio in HCC cells (Fig. 5E and 5F). These data demonstrate that sCLU might regulate the PI3K/AKT pathway by influencing the expression of Gadd45a with an involvement of tumor cell drug resistance.

\section{Discussion}

HCC is a complex disease with multiple underlying pathogenic mechanisms caused by a series of risk factors such as viral hepatitis, alcohol, and non-alcoholic steatohepatitis[18, 19]. Owing to the limit of detection and lack of health checks, $40 \%$ of patients were diagnosed with advanced liver cancer with a loss of the opportunity for operation. These patients can only be treated with local ablation therapy, radiofrequency ablation therapy, hepatic arterial chemoembolization, targeting therapy and biotherapy[20, 21]. Although systemic chemotherapy 
was used in treating HCC in the advanced stage, the clinical application of chemotherapy in HCC remains stagnant because of low sensitivity to chemotherapy, abundant side effects and weak liver functions. Complications of cirrhosis, including deranged liver function, esophageal varices and thrombocytopenia, have rendered a significant number of patients unsuitable for chemotherapy and targeted therapy. Identification of novel targets through molecular cell biology will provide new therapeutic strategies for advanced-stage HCC and better methods for outcome prediction.

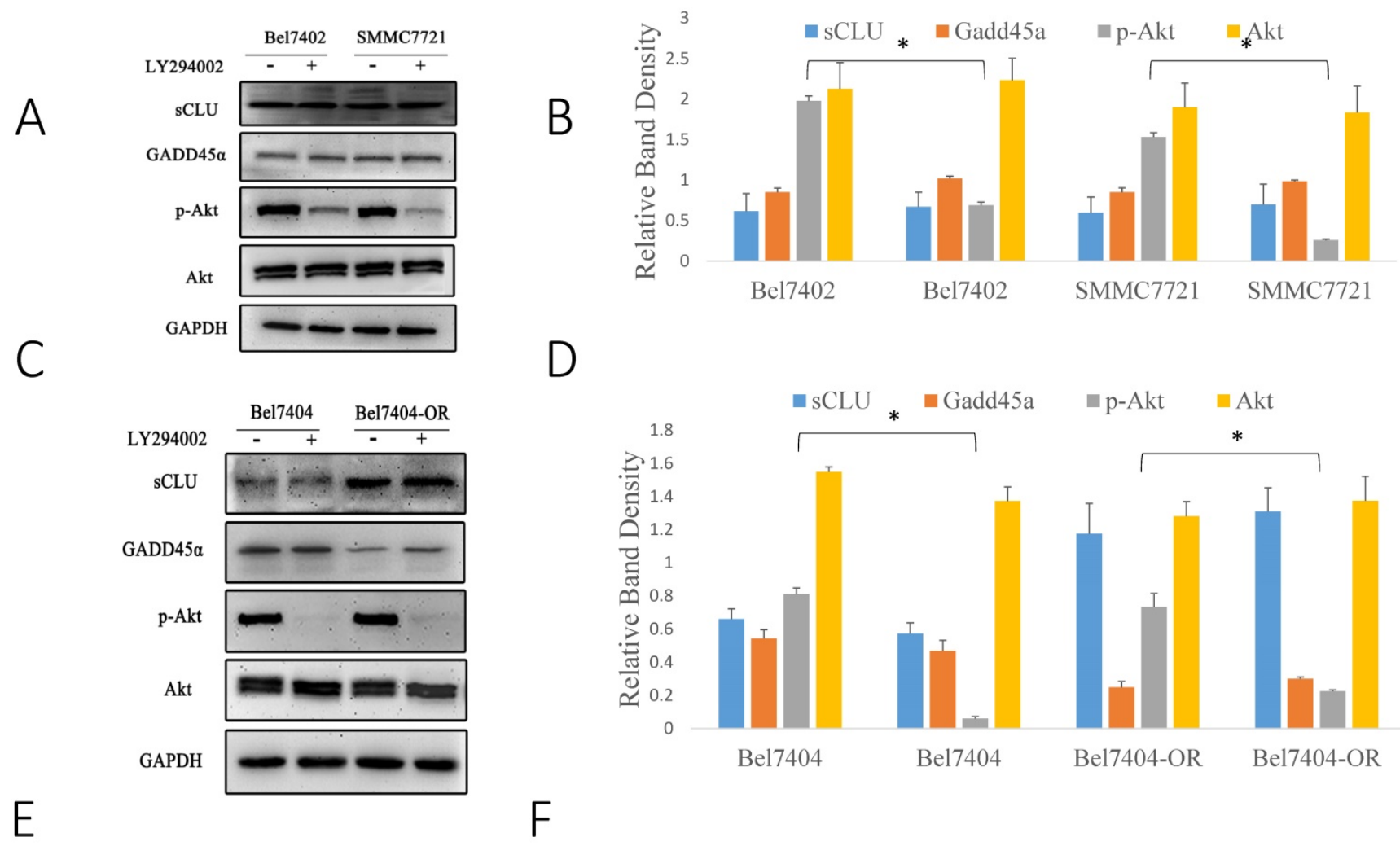

Bel7402 SMMC7721 Bel7404-OR

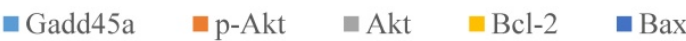
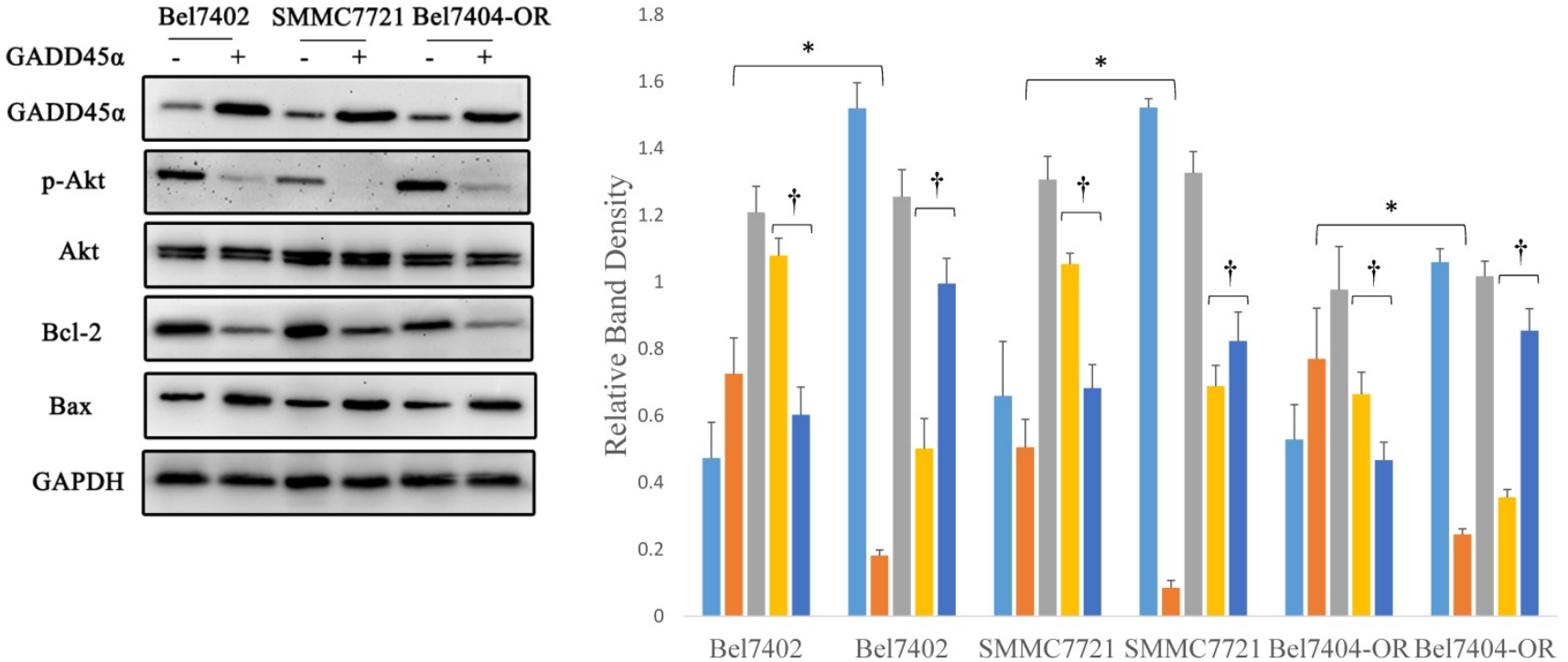

Fig. 5. sCLU regulates the PI3K/AKT signaling pathway by mediating the expression of Gadd45a. (A, C) Bel7402, SMMC7721, Bel7404 and Bel7404-OR cells were cultured in medium with the Akt inhibitor (LY294002) for $2 \mathrm{~h}$ and then were cultured in the nature medium. Western blot analysis with anti-sCLU, anti-Gadd45a, anti-phospho-Akt and anti-Akt antibody was performed to detect the protein expression. (E) Bel7402, SMMC7721 and Bel7404-OR cells, or cells transfected with pcDNA3.1-Gadd45a-His vector, were cultured in medium. Western blot analysis was done to detect the expression. (B, D, F) The density of each band in (A, C and E) was measured and normalized to that of GAPDH. A significant $(\mathrm{P}<0.05)$ difference in $\mathrm{P}$-Akt activation compared with the respective control is denoted by “*”, and the Bcl-2/Bax ratio is denoted by " $\nmid$ ". 


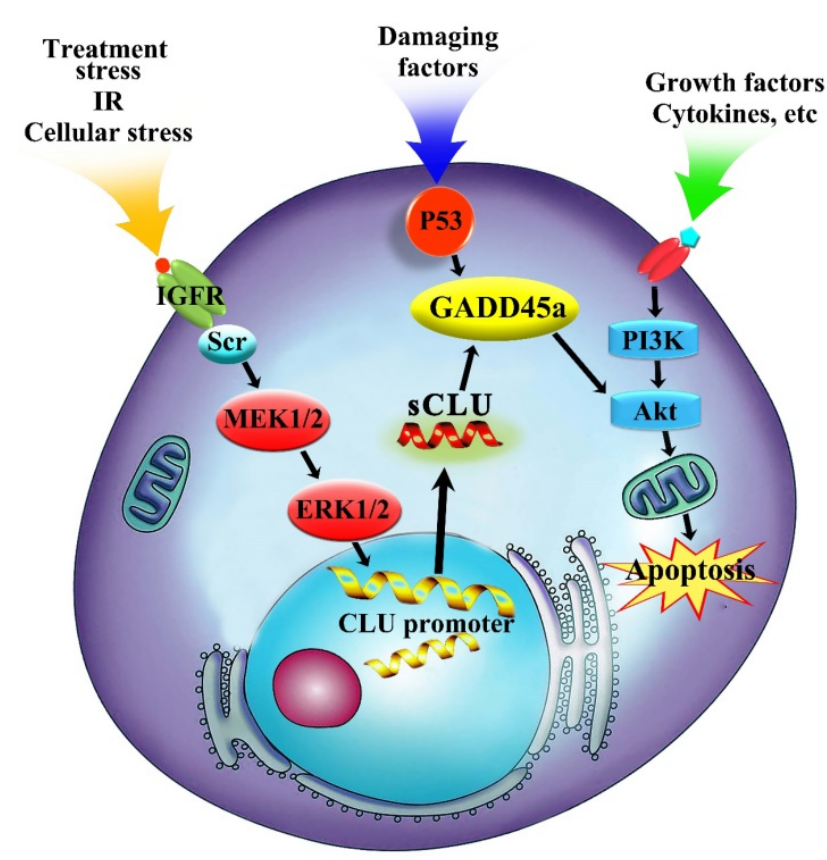

Fig. 6. Regulation mechanism of sCLU in tumor progression. The $s C L U$ promoter is activated by treatment stress or cellular stress, and then, sCLU is upregulated by activation of the Scr-MEK-ERK signaling pathway. Downregulation of sCLU inhibits the expression of Gadd45a and further decreases the p-AKT level.

Consistent with the findings of a previous study, sCLU expression was significantly higher in tumor tissues compared with the matched adjacent non-tumor tissues in a broad range of human cancers, such as breast cancer[22], lung cancer[23], and especially HCC[14, 24-26]. Likewise, the prognostic significance of sCLU expression has been reported in different tumor types. Additionally, the expression level of sCLU in some tumors was found to be closely associated with the pathological stage. The overall survival rate of patients with positive sCLU expression was significantly lower than that of patients with negative expression[15, 27, 28]. In our previous study, we found that sCLU expression is significantly increased in HCC tissues compared with that in adjacent non-tumorous liver tissues. Moreover, high sCLU expression was significantly associated with poor differentiation and advanced TNM stage[14]. However, the relationship between sCLU expression and overall survival rate in HCC patients was not analyzed. In the following study, Wang et al[29] analyzed sCLU expression using tissue microarray by immunochemistry analysis. They found that the upregulation of sCLU predicts a poor prognosis in HCC patients. Recently, similar conclusions have also been confirmed in another HCC study[30]. However, the relationship between sCLU expression and survival prognosis still needs to be confirmed in multiple research centers considering the possibility that sCLU may become an important target in HCC treatment. In this study, the conclusion that sCLU expression was significantly upregulated in HCC tissues was the same as that in our previous studies[14]. Moreover, negative sCLU expression was associated with significantly better survival rates, while sCLU was an independent prognostic factor for overall survival. These findings indicate that the assessment of sCLU expression may aid in the determination of the prognosis of patients with HCC.

sCLU, as an important anti-apoptotic factor, was considered to be involved in chemosensitivity or radiosensitivity in many human tumors[31, 32]. In our previous research, a series of genes downstream of sCLU in the apoptosis signaling pathway was discovered, and Gadd45a may be an important regulating target in the response to cell-deathinducing stimuli[14]. However, the role of Gadd45a in the chemosensitization to OXA in HCC remains unclear. Aberrant expression of Gadd45a after regulating sCLU in HCC cells has been detected (Fig. 2 ), and whether Gadd45a plays a key role in tumor drug resistance is an interesting question. Therefore, we decreased the expression of sCLU and increased the expression of Gadd45a to elucidate its direct role in the chemosensitivity of HCC cells to OXA by the CCK-8 assay and flow cytometry (Fig. 3 and 4). OXA alone significantly reduced the viability of HCC cells, whereas the depletion of sCLU and overexpression of Gadd45a significantly decreased the viability of cells treated with OXA. The same experiments were also carried out in OXA-resistant HCC cells (Bel7404-OR). The above data clearly demonstrate that the altered level of Gadd45a was responsible for changes in the drug-resistant phenotypes of human HCC cells. In other words, Gadd45a may mediate tumor drug resistance in HCC cells. This was consistent with previous studies. Downregulating the expression of sCLU can enhance the cytotoxicity of various chemotherapeutic agents[22, 33].

The PI3K/AKT signaling pathway, which can phosphorylate multiple substrates and downstream effectors such as members of the Forkhead family, caspase family, cell cycle protein family and nuclear factor- $\mathrm{kB}$, is one of the most important signaling pathways in the control of multiple biological processes in tumor cells[34, 35]. The PI3K/AKT signaling pathway can be overactivated by enhanced stimulation of receptor tyrosine kinases, particularly the IGFR and EGFR, which are upregulated in HCC and result in the stimulation of the PI3K/AKT signaling pathway[12]. In addition, many small molecule inhibitors of the PI3K/AKT signaling pathway could increase the chemosensitivity of tumor cells to anticancer agents[35]. Current research suggests that Gadd45a was shown to be a target of the p53 homologues and the expression of Gadd45a is 
known to be regulated by both p53-dependent and p53-independent pathways depending on the type of genotoxic stress[36]. Upregulation of Gadd45a is an essential step for apoptosis induction in cancer cells by various pro-apoptotic agents[37-39]. In the present research, specific inhibition of Akt could significantly decrease the phosphorylation level of Akt but did not affect the expression of sCLU and Gadd45a in HCC cells. The effect of Gadd45a on the PI3K/AKT signaling pathway is also supported by its regulatory effects on Bcl-2 family members. The altered expression of Gadd45a markedly modulated the activity of the PI3K/AKT signaling pathway in HCC cells. These results indicated that sCLU mediated HCC drug resistance to OXA through Gadd45a and then, at least in part, regulated the PI3K/AKT signaling pathway (Fig. 6).

Altogether, we provide insight into the biological function of sCLU signaling in HCC and demonstrate that the downregulated expression of sCLU restrains the activation of the PI3K/AKT pathway by upregulating the expression of Gadd45a, thus leading to enhanced chemosensitivity to OXA in HCC cells. Although we have found that sCLU induces drug resistance to OXA and elucidated its segmental molecular mechanisms in HCC cells, other potential mechanisms may still be unidentified. Therefore, the downstream molecular mechanisms of Gadd45a and PI3K/AKT signaling pathway in drug resistance in HCC should be further investigated. The new therapeutic target in combination with conventional chemotherapeutics may contribute to potential novel therapeutic methods in HCC.

\section{Abbreviations}

HCC: hepatocellular carcinoma; HBV: hepatitis $B$ virus; PI3K: phosphoinositide3-Kinase; AKT: protein kinase B; p-AKT: phosphorylated AKT; sCLU: secretory clusterin; OXA: oxaliplatin; GAPDH: glyceraldehyde-3-phosphate dehydrogenase

\section{Acknowledgements}

This research was supported by Shandong Provincial Natural Science Foundation, China (ZR2015HL080 and ZR2015HL079), Shandong Provincial Medicine and Health Science Technology Development Planning (2015WS0228) and Shandong Provincial Science and Technology Development Planning, China (2015GSF121040).

\section{Ethical approval}

This study was performed in accordance with the ethical standards as laid down in the 1964 Declaration of Helsinki and its later amendments. This study was also approved by the research Ethics
Committee of Shandong Provincial Qianfoshan Hospital.

\section{Competing Interests}

The authors have declared that no competing interest exists.

\section{References}

1. Chen $\mathrm{W}$, Zheng $\mathrm{R}$, Zeng $\mathrm{H}$, Zhang $\mathrm{S}$. The incidence and mortality of major cancers in China, 2012. Chinese journal of cancer. 2016; 35: 73.

2. Torre LA, Bray F, Siegel RL, Ferlay J, Lortet-Tieulent J, Jemal A. Global cancer statistics, 2012. CA: a cancer journal for clinicians. 2015; 65: 87-108.

3. Wilhelm S, Carter C, Lynch M, Lowinger T, Dumas J, Smith RA, et al. Discovery and development of sorafenib: a multikinase inhibitor for treating cancer. Nature reviews Drug discovery. 2006; 5: 835-44.

4. Xie B, Wang DH, Spechler SJ. Sorafenib for treatment of hepatocellular carcinoma: a systematic review. Digestive diseases and sciences. 2012; 57: $1122-9$.

5. Xiu P, Dong XF, Li XP, Li J. Clusterin: Review of research progress and looking ahead to direction in hepatocellular carcinoma. World journal of gastroenterology. 2015; 21: 8262-70.

6. Mukherji D, Eichholz A, De Bono JS. Management of metastatic castration-resistant prostate cancer: recent advances. Drugs. 2012; 72: 1011-28.

7. Rodriguez-Pineiro AM, Garcia-Lorenzo A, Blanco-Prieto S, Alvarez-Chaver P, Rodriguez-Berrocal FJ, Cadena MP, et al. Secreted clusterin in colon tumor cell models and its potential as diagnostic marker for colorectal cancer. Cancer investigation. 2012; 30: 72-8.

8. Wilson MR, Zoubeidi A. Clusterin as a therapeutic target. Expert opinion on therapeutic targets. 2017; 21: 201-13

9. Saad F, Hotte S, North S, Eigl B, Chi K, Czaykowski P, et al. Randomized phase II trial of Custirsen (OGX-011) in combination with docetaxel or mitoxantrone as second-line therapy in patients with metastatic castrate-resistant prostate cancer progressing after first-line docetaxel: CUOG trial P-06c. Clinical cancer research : an official journal of the American Association for Cancer Research. 2011; 17: 5765-73.

10. Laskin JJ, Nicholas G, Lee C, Gitlitz B, Vincent M, Cormier Y, et al. Phase I/II trial of custirsen (OGX-011), an inhibitor of clusterin, in combination with a gemcitabine and platinum regimen in patients with previously untreated advanced non-small cell lung cancer. Journal of thoracic oncology : official publication of the International Association for the Study of Lung Cancer. 2012; 7: 579-86.

11. Beer TM, Hotte SJ, Saad F, Alekseev B, Matveev V, Flechon A, et al. Custirsen (OGX-011) combined with cabazitaxel and prednisone versus cabazitaxel and prednisone alone in patients with metastatic castration-resistant prostate cancer previously treated with docetaxel (AFFINITY): a randomised, open-label, international, phase 3 trial. The Lancet Oncology. 2017.

12. Whittaker $S$, Marais R, Zhu AX. The role of signaling pathways in the development and treatment of hepatocellular carcinoma. Oncogene. 2010; 29: 4989-5005.

13. Xiu P, Dong $X$, Dong $X, X u Z$, Zhu H, Liu F, et al. Secretory clusterin contributes to oxaliplatin resistance by activating Akt pathway in hepatocellular carcinoma. Cancer science. 2013; 104: 375-82.

14. Xiu P, Xu Z, Liu F, Li Z, Li T, Zou F, et al. Downregulating sCLU enhances the sensitivity of hepatocellular carcinoma cells to gemcitabine by activating the intrinsic apoptosis pathway. Digestive diseases and sciences. 2014; 59: 1798-809.

15. Watari H, Ohta Y, Hassan MK, Xiong Y, Tanaka S, Sakuragi N. Clusterin expression predicts survival of invasive cervical cancer patients treated with radical hysterectomy and systematic lymphadenectomy. Gynecologic oncology. 2008; 108: 527-32.

16. Miyake H, Muramaki M, Kurahashi T, Yamanaka K, Hara I, Gleave M, et al. Expression of clusterin in prostate cancer correlates with Gleason score but not with prognosis in patients undergoing radical prostatectomy without neoadjuvant hormonal therapy. Urology. 2006; 68: 609-14.

17. Henderson-Jackson EB, Nasir A, Chen DT, Nandyala P, Djeu J, Strosberg J, et al. Cytoplasmic Clusterin expression correlates with pancreatic neuroendocrine tumor size and pathological stage. Pancreas. 2013; 42: 967-70.

18. Cholankeril G, Patel R, Khurana S, Satapathy SK. Hepatocellular carcinoma in non-alcoholic steatohepatitis: Current knowledge and implications for management. World journal of hepatology. 2017; 9: 533-43.

19. Shimizu D, Inokawa Y, Sonohara F, Inaoka K, Nomoto S. Search for useful biomarkers in hepatocellular carcinoma, tumor factors and background liver factors (Review). Oncology reports. 2017; 37: 2527-42.

20. Cidon EU. Systemic treatment of hepatocellular carcinoma: Past, present and future. World journal of hepatology. 2017; 9: 797-807.

21. Nishikawa H, Kimura T, Kita R, Osaki Y. Treatment for hepatocellular carcinoma in elderly patients: a literature review. Journal of Cancer. 2013; 4: 635-43. 
22. So A, Sinnemann S, Huntsman D, Fazli L, Gleave M. Knockdown of the cytoprotective chaperone, clusterin, chemosensitizes human breast cancer cells both in vitro and in vivo. Molecular cancer therapeutics. 2005; 4: 1837-49.

23. Li H, Liu S, Zhu X, Yang S, Xiang J, Chen H. Clusterin immunoexpression and its clinical significance in patients with non-small cell lung cancer. Lung. 2010; 188: 423-31.

24. Kang YK, Hong SW, Lee H, Kim WH. Overexpression of clusterin in human hepatocellular carcinoma. Human pathology. 2004; 35: 1340-6.

25. Lau SH, Sham JS, Xie D, Tzang CH, Tang D, Ma N, et al. Clusterin plays an important role in hepatocellular carcinoma metastasis. Oncogene. 2006; 25: 1242-50.

26. Wang C, Jiang K, Gao D, Kang X, Sun C, Zhang Q, et al. Clusterin protects hepatocellular carcinoma cells from endoplasmic reticulum stress induced apoptosis through GRP78. PloS one. 2013; 8: e55981.

27. Kruger S, Ola V, Fischer D, Feller AC, Friedrich M. Prognostic significance of clusterin immunoreactivity in breast cancer. Neoplasma. 2007; 54: 46-50.

28. Hassan MK, Watari H, Han Y, Mitamura T, Hosaka M, Wang L, et al. Clusterin is a potential molecular predictor for ovarian cancer patient's survival: targeting clusterin improves response to paclitaxel. Journal of experimental \& clinical cancer research : CR. 2011; 30: 113.

29. Wang C, Jin G, Jin H, Wang N, Luo Q, Zhang Y, et al. Clusterin facilitates metastasis by EIF3I/Akt/MMP13 signaling in hepatocellular carcinoma. Oncotarget. 2015; 6: 2903-16.

30. Zheng W, Yao M, Qian Q, Sai W, Qiu L, Yang J, et al. Oncogenic secretory clusterin in hepatocellular carcinoma: Expression at early staging and emerging molecular target. Oncotarget. 2017; 8: 52321-32.

31. Zellweger T, Chi K, Miyake H, Adomat H, Kiyama S, Skov K, et al. Enhanced radiation sensitivity in prostate cancer by inhibition of the cell survival protein clusterin. Clinical cancer research : an official journal of the American Association for Cancer Research. 2002; 8: 3276-84.

32. Gleave M, Chi KN. Knock-down of the cytoprotective gene, clusterin, to enhance hormone and chemosensitivity in prostate and other cancers. Annals of the New York Academy of Sciences. 2005; 1058: 1-15.

33. Hoeller C, Pratscher B, Thallinger C, Winter D, Fink D, Kovacic B, et al. Clusterin regulates drug-resistance in melanoma cells. The Journal of investigative dermatology. 2005; 124: 1300-7

34. Lin $X$, Zhang $X$, Wang $Q$, Li J, Zhang $P$, Zhao $M$, et al. Perifosine downregulates MDR1 gene expression and reverses multidrug-resistant phenotype by inhibiting PI3K/Akt/NF-kappaB signaling pathway in a human breast cancer cell line. Neoplasma. 2012; 59: 248-56.

35. Chen IC, Hsiao LP, Huang IW $\mathrm{Yu} \mathrm{HC}_{\text {, }} \mathrm{Yeh} \mathrm{LC}_{\text {, }} \mathrm{Lin} \mathrm{CH}_{\text {, et al. }}$ Phosphatidylinositol-3 Kinase Inhibitors, Buparlisib and Alpelisib, Sensitize Estrogen Receptor-positive Breast Cancer Cells to Tamoxifen. Scientific reports. 2017; 7: 9842.

36. Rosemary Siafakas A, Richardson DR. Growth arrest and DNA damage- 45 alpha (GADD45alpha). The international journal of biochemistry \& cell biology. 2009; 41: 986-9.

37. Yang C, Yang S, Wood KB, Hornicek FJ, Schwab JH, Fondren G, et al. Multidrug resistant osteosarcoma cell lines exhibit deficiency of GADD45alpha expression. Apoptosis : an international journal on programmed cell death. 2009; 14: 124-33.

38. Jiang T, Soprano DR, Soprano KJ. GADD45A is a mediator of CD437 induced apoptosis in ovarian carcinoma cells. Journal of cellular physiology. 2007; 212: 771-9.

39. Smith GB, Mocarski ES. Contribution of GADD45 family members to cell death suppression by cellular Bcl-xL and cytomegalovirus vMIA. Journal of virology. 2005; 79: 14923-32. 